\title{
Effects of blocking of the avoidance response on the elimination of the conditioned fear response'
}

TERRY A. BENLIHE AND EDWARD C. SIMMEL MIAMI UNIVERSITY

The effects of fear extinction on the subsequent extinction of the instrumental avoidance response was investigated in the albino rat. Statistical analyses and graphic representation revealed that blocked $S s$ gave fewer responses during extinction than controls, this difference diminishing as a function of the number of extinction trials. Latency results were supportive. The authors concluded that blocking of the avoidance response in the presence of conditioned fear producing stimuli results in only a temporary suppression of the avoidance behavior.

Various studies have shown that fear, once acquired, can elicit avoidance behavior over relatively long periods of time, and that both these fears and the avoidance behaviors are unaffected by usual laboratory extinction procedures (Moyer, 1958; Solomon, Kamin, \& Wynne, 1952).

Mowrer (1951) has suggested that there are two distinct learning processes-the classical conditioning of fear and the instrumental conditioning of the avoidance response-occurring in the acquisition of an avoidance habit. Solomon \& Wynne (1954), following Mowrer's paradigm, proposed the anxiety conservation hypothesis to explain the relatively greater persistence of habits acquired under aversive stimulation. The present study was based upon two predictions derived from these theories: (1) If there are two distinct learning processes in avoidance learning, then one should be able to extinguish one process (fear) separately, and by doing so facilitate extinction of the other (hurdle-jumping); (2) If the anxiety conservation hypothesis is correct, then blocking the animal in the presence of fear-provoking situations should allow for the full fear reaction to occur and consequently result in its extinction. Further, the extinction of fear should facilitate extinction of the instrumental avoidance response.

Method

The experiment was divided into three phases: (1) A training phase, in which rats were conditioned to avoid a light; (2) a treatment phase, consisting of blocking animals in the presence of light; and (3) a normal extinction procedure. There were two replications of the study with 16 Ss serving in each. During the training phase, all Ss received 50 training trials in a shuttlebox, divided at the center by a 5 in.hurdle. A light preceded shock by $3 \mathrm{sec}$ and both light and shock terminated when the animal crossed the hurdle. Following training, for the treatment phase, Ss were divided into four groups of four rats in each group. Groups were matched on sex and the number of avoidance responses given on the last 20 training trials. Group 1, the nominal control, received no trials during the blocking phase, while Groups 2, 3, and 4 received eight, 16, and 32 trials/day, respectively, over a five day period. With a glass barrier in the center of the apparatus and flush with the hurdle to prevent Ss from making an avoidance response, the CS was presented by itself for $20 \mathrm{sec}$ on each of the blocking trials. After blocking, all Ss were given 100 extinction trials over a five day period.

\section{Results and Discussion}

On early extinction trials experimental animals gave fewer responses than controls and responded with longer latencies. However, the degree to which groups differed diminished as a function of blocks of extinction trials (see Figs. 1 and 2). By the last block of 20 trials, extinction performance was comparable for all groups. Statistical analysis of the original experiment strongly supported the graphic representation. F-tests on simple effects for group responses over each separate extinction day revealed changes in probability levels consistent with the graphed data: Day 1, $p<.01$; Day $2, \mathrm{p}<$ .10; Day 3, $<<.025$; Days 4 and 5 , no difference $(\mathrm{df}=$ $3 / 12)$. A corresponding analysis of the replication experiment supported in general that cited above. Graphic representation of the data indicated the same general trends, although greater variability yielded somewhat different statistical results. The tests on simple main effects were needed because of large interaction terms in the overall groups by days analysis. This was true

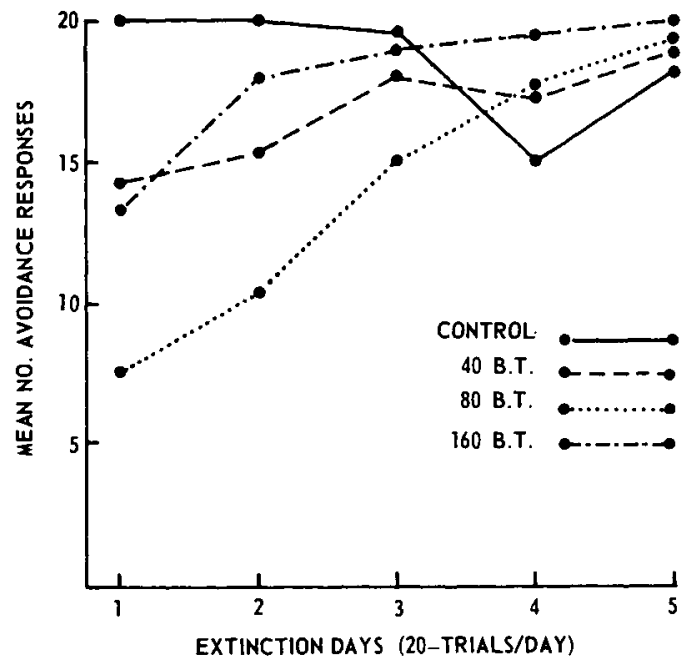

Fig. 1. Blocked trial (BT) groups' and control group's mean responses over days. 


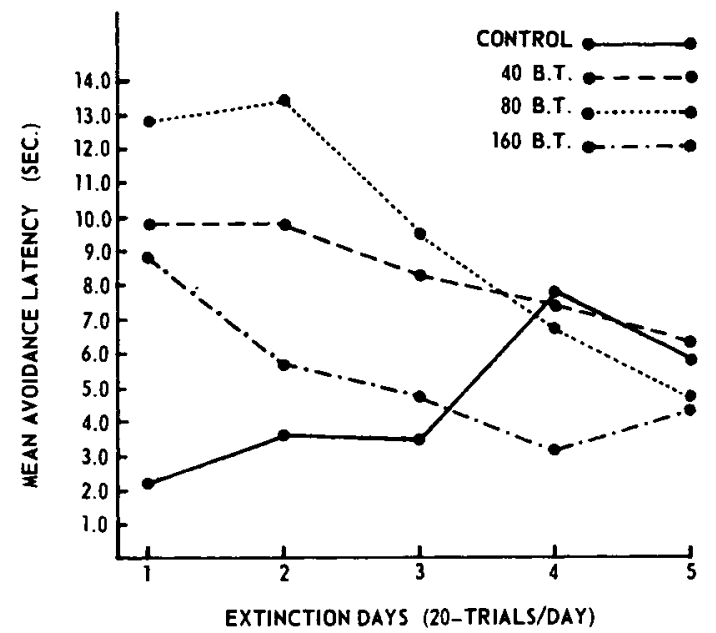

Fig. 2. Mean avoidance latencies of blocked trials (BT) groups' and control group's responses over days.

for both response and latency measures in both experiments.

On the early extinction trials, as shown in Figs. 1 and 2, predictions derived from the theories of Mowrer (1951) and Solomon \& Wynne (1954) seem to be supported. Experimental animals, which had been blocked for varying periods of time in the presence of the fear provoking situation, gave fewer responses and responded with greater latencies than control animals. However, these differences disappeared rapidly and by the fifth day, at the end of 100 extinction trials, all animals were giving approximately the same number of responses and responding with similar latencies. It may be noted in the figures that during extinction sessions the control group gave successively fewer responses which increased in latency, while experimental groups responded with successively greater frequency which decreased in latency. In some cases these animals exhibited performances at the end of extinction which equaled their response levels at the end of training. Apparently blocking in the presence of the CS alone only temporarily suppressed the instrumental habit and had little or no permanent effect on the extinction of fear.

It is possible that the effect of the blocking treatment was to facilitate the learning of a response incompatible with hurdle-jumping. Records which were kept on the behavior of Ss at the time of CStermination showed that in no case had an animal learned a new response (i.e., crouching) which would interfere with hurdle-jumping.
A second possible interpretation of the temporary suppression was that Ss were punished during the blocking phase. Several animals did in fact jump into the barrier, but this occurred only in the first treatment day and never more than twice. Furthermore, the barrier was clearly discriminable to the animals and those who did jump into it seemed to do so with great caution and little force.

Observationally, it appears as if the temporary suppression is due to "confusion" on the part of experimental Ss. During training, the light went off only when $S$ crossed the hurdle to the "safe side" of the apparatus. However, during the blocking phase, $S$ could not escape and the CS always terminated while he was in the starting compartment. "Confusion" ended for most animals with the first successful avoidance response. Typically, response levels increased and latencies dropped radically following the first response.

Three reasons are postulated for this sudden change in behavior. (1) The first jumping response seemed to re-integrate the original learning situation. Jumping should have been more specifically associated with light termination than were the numerous and general behaviors exhibited during the blocking phase. Also, jumping should have been the most dominant response having been learned to the primary reinforcement of shock. (2) During extinction, Ss could terminate the light as quickly as they could make the jumping response rather than waiting $20 \mathrm{sec}$ for the trial to end. Thus, if the aversive properties of the CS had not been completely extinguished during Phase 2, immediate reinforcement could be achieved by immediate jumping. (3) If the animal did jump, he eliminated not only the light but also all other conditioned aversive properties associated with the shock side of the box.

\section{References}

Mowrer, o. H. Two-factor learning theory: summary and comment. Psychol. Rev., 1951, 58, 350-354.

Moyer, K. E. Effect of delay between training and extinction on the extinction of an avoidance response. J. comp. physiol. Psychol. 1958, 51, 116-118.

Solomon, R. L., \& Wynne, L. C. Traumatic avoidance learning: the principles of anxiety conservation and partial irreversibility. Psychol. Rev., 1954, 61, 353-385.

Solomon, R. L., Kamin. L. J.. \& Wynne. L. C. Traumatic avoidance learning: the outcomes of several extinction procedures with dogs. J. abnorm. soc. Psychol., 1952, 48, 291-302.

\section{Note}

1. This study is based on data collected as part of the M. A. Thesis of the first author, under the supervision of the second author at Miami University, Oxford, Ohio. It was read in part at the Midwestern Psychological Association meeting, Chicago, 1967. 